Published in final edited form as:

Science. 2012 March 2; 335(6072): 1055-1056. doi:10.1126/science.1219302.

\title{
CELL SIGNALING:
}

\section{Structural Origins of Receptor Bias}

\author{
Stephen R. Sprang and Jackson Chief Elk \\ Center for Biomolecular Structure and Dynamics, University of Montana, Missoula, MT 59812, \\ USA
}

Stephen R. Sprang: stephen.sprang@umontana.edu

\section{Abstract}

Agonists can elicit pathway-specific conformational changes in a G protein-coupled receptor.

Humans express more than $800 \mathrm{G}$ protein-coupled receptors (GPCRs), through which myriad physiological, immune, and neurophysiological processes are regulated (1). These receptors are embedded in the cell's plasma membrane and bind to extracellular chemical stimuli (agonists). This interaction induces conformational changes in the receptor that converge at intracellular sites that bind to either heterotrimeric guanine nucleotide-binding proteins ( $\mathrm{G}$ proteins) or to $\beta$-arrestin proteins that block $\mathrm{G}$ protein binding and lead to receptor desensitization and internalization, as well as provide a scaffold for protein regulatory complexes that control gene expression (2). Many agonists induce functionally selective or biased states of the receptor, wherein one pathway is activated with greater efficacy than another (3). Thus, GPCRs are not simple on-off switches, but can adopt multiple conformational states to control diverse processes. On page 1106 of this issue, Liu et al. (4) provide clues as to how a GPCR called the $\beta_{2}$-adrenergic receptor ( $\beta_{2} \mathrm{AR}$ ) can tune its conformation to achieve a balance of functional outcomes, as directed by the chemical structure of the receptor agonist.

Like all GPCRs, the polypeptide chain of the $\beta_{2} \mathrm{AR}$ traverses back and forth across the membrane, forming a series of seven-transmembrane (TM) a helices (see the figure). Ligands occupy a site that is deep within the receptor but accessible to the extracellular environment. Adrenergic receptor agonists are typically composed of a cyclic aromatic "head" group and an alkyl-amine "tail." Crystal structure analyses of the $\beta_{1} A R$ and $\beta_{2} A R$ reveal that the head group reinforces contacts between TM5 and TM6, whereas the amine substituent stabilizes interactions between TM3 and TM7 $(5,6)$. Conformational changes upon ligand binding follow a common theme in the three activated GPCRs, including the $\beta_{2} \mathrm{AR}$, that have so far been crystallized (7-9). Local structural perturbations induced by agonist binding are transduced into a concerted outward displacement of TM5 and TM6, which opens a pocket on the intracellular surface of the receptor that accepts the $G$ protein. Binding of $\beta$-arrestin to GPCRs requires the addition of phosphate groups at specific sites on the intracellular surface of the receptor by GPCR protein kinases. This phosphorylation occurs at a separate site, beyond TM7, toward the C terminus of the receptor. Structural changes at the intracellular side of TM6 and TM7 would therefore be diagnostic of ligands that show selective agonism toward $G$ protein or $\beta$-arrestin signaling pathways.

To monitor the structural consequences of agonist binding, Liu et al. attached fluorine-19 $\left({ }^{19} \mathrm{~F}\right)$ probes to cysteine residues at the intracellular ends of TM6 and TM7. The nuclear magnetic resonance (NMR) spectrum of ${ }^{19} \mathrm{~F}$ is sensitive to its chemical environment and to the dynamic behavior of the site to which it is attached. The spectra revealed that TM6 and TM7 are each in equilibrium between two distinct conformations in the basal, ligand-free 
state of the receptor. By correlating the relative population in each state with agonist potency, the authors could assign each spectral component to either the active or inactive state for $\mathrm{G}$ protein and for $\beta$-arrestin pathway activation.

Most ligands induced roughly equivalent shifts toward activating TM6 or TM7 according to their relative potency. A particularly informative antagonist, carvedilol, induced a strong shift toward the TM7 active state but had little effect on the conformational equilibrium at TM6. This antagonist not only blocks $\mathrm{G}$ protein signaling but also induces receptor desensitization and internalization (10). Drugs such as carvedilol are prototypes for biased agonists that focus signaling along a specific pathway. Site-specific NMR probes like those used by Liu et al. provide direct structural assays to guide the design of such functionally specific ligands.

Fluorescence studies performed in the past decade had already revealed a hierarchy of discrete structural states that can be induced by agonists of varying potency (partial agonists) (11-13). The weakest agonists loosen interactions (the "ionic lock" and "toggle") that hold TM3 and TM6 in the inactive state-sufficient for coupling the receptor to a G protein. Receptor interactions with the polar substituents of the aromatic "head" group of the ligand are critical for this transduction pathway. More potent agonists also perturb the Asn-Pro-X-XTyr (NPxxY, where " $x$ " is any amino acid) sequence of TM7 that permits phosphorylation of the receptor and subsequent $\beta$-arrestin binding. Substituents appended to the hydroxylamine "tail" of the ligand influence its ability to activate this pathway, large nonpolar groups being particularly effective. Supercomputers "hardwired" for molecular dynamics calculations have been used to simulate multimicrosecond trajectories of the $\beta_{2} \mathrm{AR}$ in the membrane (14). These calculations provide structural insight into spectroscopically observed intermediate agonist-bound states in which the ionic lock is broken while the transition in the NPxxY motif has not yet occurred. That certain agonists can stimulate $\beta$ arrestin, but not $G$ protein signaling, suggests parallel rather than hierarchical modes of activation. Nevertheless, how any particular mode of agonist-receptor interaction stabilizes a unique conformational ensemble of the receptor is, for the most part, a mystery.

Agonist-induced receptor bias for $G$ protein versus $\beta$-arrestin signaling is one of many instances of the plastic and multistate behavior characteristic of GPCRs (15). Many receptors couple to more than one class of $\mathrm{G}$ protein, and hence can drive alternative signaling pathways, depending on the chemical structure of the agonist. Dimerization between the same or different classes of receptor signaling, and allosteric modulators that bind at loci other than the agonist site, also affect GPCR function. The challenge is to construct computational models that account for the specific effects of ligands-and of other protein partners in the membrane-on the energetic landscape of receptor conformational states, to predict how these will translate into specific biological outcomes.

\section{References}

1. Rosenbaum DM, et al. Nature. 2009; 459:356. [PubMed: 19458711]

2. Shenoy SK, Lefkowitz RJ. Trends Pharmacol. Sci. 2011; 32:521. [PubMed: 21680031]

3. Urban JD, et al. J. Pharmacol. Exp. Ther. 2007; 320:1. [PubMed: 16803859]

4. Liu JJ, et al. Science. 2012; 335:1106. [PubMed: 22267580]

5. Warne T, et al. Nature. 2011; 469:241. [PubMed: 21228877]

6. Wacker D, et al. J. Am. Chem. Soc. 2010; 132:11443. [PubMed: 20669948]

7. Scheerer P, et al. Nature. 2008; 455:497. [PubMed: 18818650]

8. Xu F, et al. Science. 2011; 332:322. [PubMed: 21393508]

9. Rasmussen SGF, et al. Nature. 2011; 477:549. [PubMed: 21772288]

10. Wisler JW, et al. Proc. Natl. Acad. Sci. U.S.A. 2007; 104:16657. [PubMed: 17925438] 
11. Ghanouni P, et al. J. Biol. Chem. 2001; 276:24433. [PubMed: 11320077]

12. Swaminath G, et al. J. Biol. Chem. 2005; 280:22165. [PubMed: 15817484]

13. Yao X, et al. Nat. Chem. Biol. 2006; 2:417. [PubMed: 16799554]

14. Dror RO, et al. Proc. Natl. Acad. Sci. U.S.A. 2011; 108:18684. [PubMed: 22031696]

15. Kenakin T, Miller LJ. Pharmacol. Rev. 2010; 62:265. [PubMed: 20392808] 
Extracellular environment
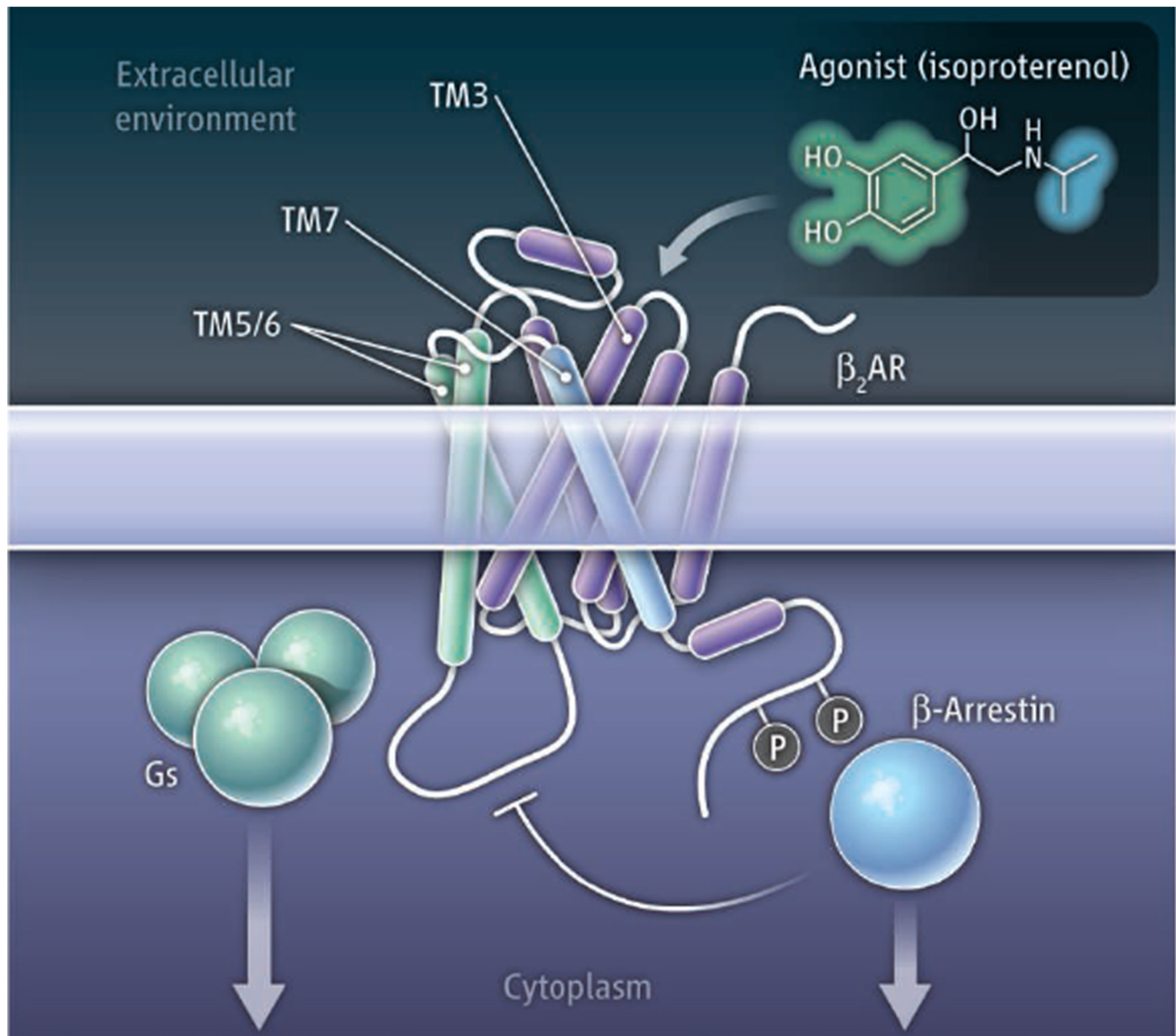

G protein signaling pathways

$\beta$-Arrestin signaling pathways

Figure 1. Functionally selective

Binding of agonists to the $\beta_{2} \mathrm{AR}$ results in conformational changes that displace TM5 and TM6 (green) and/or TM7 (blue). The conformational changes permit G protein (Gs) binding or receptor phosphorylation $(\mathrm{P})$ and $\beta$-arrestin binding. $\beta$-arrestin binding blocks $\mathrm{G}$ protein signaling. The aromatic moiety of the agonist (isoproterenol shown) contacts TM5 and TM6, whereas the hydroxylamine substituent interacts with TM3 and TM7. 